\title{
ON THE SOURCE OF PROPAGATING SLOW MAGNETOACOUSTIC WAVES IN SUNSPOTS
}

\author{
S. Krishna Prasad ${ }^{1}$, D. B. Jess ${ }^{1,2}$, and Elena Khomenko ${ }^{3}$ \\ ${ }^{1}$ Astrophysics Research Centre, School of Mathematics and Physics, Queen's University Belfast, Belfast BT7 1NN, UK; krishna.prasad@qub.ac.uk \\ ${ }^{2}$ Department of Physics and Astronomy, California State University Northridge, Northridge, CA 91330, USA \\ ${ }^{3}$ Instituto de Astrofísica de Canarias, E-38205 La Laguna, Tenerife, Spain \\ Received 2015 August 15; accepted 2015 September 17; published 2015 October 9
}

\begin{abstract}
Recent high-resolution observations of sunspot oscillations using simultaneously operated ground- and spacebased telescopes reveal the intrinsic connection between different layers of the solar atmosphere. However, it is not clear whether these oscillations are externally driven or generated in situ. We address this question by using observations of propagating slow magnetoacoustic waves along a coronal fan loop system. In addition to the generally observed decreases in oscillation amplitudes with distance, the observed wave amplitudes are also found to be modulated with time, with similar variations observed throughout the propagation path of the wave train. Employing multi-wavelength and multi-instrument data, we study the amplitude variations with time as the waves propagate through different layers of the solar atmosphere. By comparing the amplitude modulation period in different layers, we find that slow magnetoacoustic waves observed in sunspots are externally driven by photospheric $p$-modes, which propagate upward into the corona before becoming dissipated.
\end{abstract}

Key words: magnetohydrodynamics (MHD) - sunspots - Sun: atmosphere - Sun: oscillations - Sun: photosphere

Supporting material: animation

\section{INTRODUCTION}

Waves and oscillations are an integral part of sunspots. To date, there have been many observational reports of oscillations in the photosphere, umbral flashes and running penumbral waves in the chromosphere, and propagating waves in the corona (see, e.g., the review articles by Bogdan \& Judge 2006; Jess et al. 2015; Khomenko \& Collados 2015). Oscillations manifesting in photospheric sunspots usually display prominent five-minute periodicities alongside traces of power in threeminute bandpasses, albeit with relatively small amplitudes compared to those present in the surrounding quiet Sun (Bhatnagar et al. 1972; Abdelatif et al. 1984; Landgraf 1997; Bellot Rubio et al. 2000). Umbral flashes (Beckers \& Tallant 1969) are approximately three-minute periodic brightenings in chromospheric umbrae that occur as a result of upwardly propagating magnetoacoustic oscillations converting into localized shock waves (Rouppe van der Voort et al. 2003; Henriques et al. 2015).

Running penumbral waves are outwardly propagating oscillations found in the chromospheric penumbrae of sunspots (Giovanelli 1972; Zirin \& Stein 1972). The periodicities of these waves, which are on the order of a few minutes, increase from the inner penumbra to the outer boundary, while the associated phase speeds decrease (Brisken \& Zirin 1997; Christopoulou et al. 2000; Jess et al. 2013). It is believed that the "trans-sunspot" (i.e., outward) motion is apparent to a given line of sight and that these oscillations actually represent the upward propagation of field-guided magnetoacoustic waves from the photosphere (Christopoulou et al. 2001; Kobanov et al. 2006; Bloomfield et al. 2007; Jess et al. 2013). The gradual change in inclination of the penumbral field lines produces the observed changes in the oscillation periods and phase speeds. Recently, Löhner-Böttcher \& Bello González (2015) identified the photospheric signatures of running penumbral waves and found them to be consistent with the upward propagation of magnetoacoustic waves predicted previously. Reznikova et al. (2012) have even suggested frequency fluctuations within umbrae itself indicate substantial local variations in magnetic field inclinations.

Evidence for propagating waves along fanlike loop structures in the corona is in abundance. The fan loops associated with sunspots are usually rooted in the umbra and display propagating waves with periodicities approximately equal to three minutes (De Moortel et al. 2002; Marsh \& Walsh 2006; Jess et al. 2012a; Reznikova et al. 2012). The amplitude of these waves decreases rapidly as they propagate along the loop, eventually disappearing after several thousand kilometers. Several physical processes such as thermal conduction and compressive viscosity are believed to dissipate such waves in the corona (De Moortel \& Hood 2003; Krishna Prasad et al. 2014). Jess et al. (2012a) found an association between threeminute waves propagating along fan loops and simultaneous amplitude enhancements in underlying photospheric umbral dots. Their results suggest that magnetoacoustic waves manifesting in the photosphere can propagate upward into the corona where, ultimately, the energy they carry is dissipated.

As advancements are being made in solar instrumentation and observations, it is becoming increasingly evident that all the above phenomena detected in different layers of the solar atmosphere are actually inter-connected and most likely produced by the same upwardly propagating slow magnetoacoustic waves, which present themselves according to the local physical conditions. Although it is often assumed that the photospheric $p$-modes are the ultimate source of sunspot oscillations, there is no clear evidence as to whether these oscillations are externally driven by the $p$-modes or generated in situ within the sunspots (e.g., through magnetoconvection). In this Letter, we aim to address this issue by studying temporal variations in the amplitudes of propagating slow waves observed in a coronal fan loop that were not explored before. We present the details on our observations, our analysis methods, and results in the subsequent sections and finally discuss the conclusions. 


\section{OBSERVATIONS}

The Dunn Solar Telescope at Sacramento Peak, New Mexico, was employed to observe active region NOAA 11366 on 2011 December 10 between 16:10 and 17:25 UT. The Rapid Oscillations in the Solar Atmosphere (ROSA; Jess et al. 2010) and the Hydrogen-Alpha Rapid Dynamics camera (HARDcam; Jess et al. 2012a) instruments were employed to simultaneously capture high-resolution images in four different optical channels centered at the $\mathrm{H} \alpha$ line core $(6562.8 \AA)$, Ca II K line core $(3933.7 \AA)$, G-band $(4305.5 \AA)$, and blue continuum ( $4170 \AA$ ) wavelengths, with filter bandpasses corresponding to $0.25 \AA, 1 \AA, 9.2 \AA$, and $52 \AA$, respectively. The pixel scale was 0 " 0696 for the ROSA channels, producing a field of view equal to $69^{\prime \prime} \times 69^{\prime \prime}$. For $\mathrm{H} \alpha$ images acquired by HARDcam, the plate scale was 0 !" 138 per pixel, providing a marginally larger field of view of $71^{\prime \prime} \times 71^{\prime \prime}$. Part of this data set has been used previously by Jess et al. (2013), where the authors detail the full speckle reconstruction and calibration steps applied to the data. Following all image-processing steps, the final cadences of the data are $2.11 \mathrm{~s}$ for the $4170 \AA$ continuum and G-band channels, $7.39 \mathrm{~s}$ for the $\mathrm{Ca}$ II $\mathrm{K}$ filtergrams, and $1.78 \mathrm{~s}$ for the narrowband $\mathrm{H} \alpha$ image sequence. Seeing conditions remained excellent during the 75-minute observation period. However, a few images were affected by local and short-duration atmospheric fluctuations, which resulted in slight image degradation in locations away from the adaptive optics lock point. These images, corresponding to an average duration not longer than a few seconds, were replaced through interpolation. The co-alignment between the different ROSA channels is achieved using a series of collimated targets obtained immediately after the end of the science observations.

The corresponding space-based data from the Atmospheric Imaging Assembly (AIA; Lemen et al. 2012) and the Helioseismic and Magnetic Imager (HMI; Schou et al. 2012), on board the Solar Dynamics Observatory (SDO; Pesnell et al. 2012), form the main part of the present study. Level 1.0 data from both instruments were processed using the routines aia_prep.pro and hmi_prep.pro available through standard solar software (e.g., sswiDL) pipelines. This involves bringing all the data to a common center and plate scale, with a final pixel scale $\approx 0{ }^{\prime \prime} 6$. A subfield of $210^{\prime \prime} \times 210^{\prime \prime}$ is then carefully selected (by accounting for solar rotation) around the target region from AIA 171, 131, 304, 1600, and $1700 \AA$ channels, in addition to HMI Dopplergrams covering the full observational duration. The cadence is $12 \mathrm{~s}$ for the AIA 171, 131 , and $304 \AA$ channels, 24 s for the AIA 1600 and $1700 \AA$ channels, and $45 \mathrm{~s}$ for the HMI Dopplergram data, with each image co-aligned to the first image using intensity crosscorrelation. HMI and ROSA (4170 A) continuum images were used to achieve the required co-alignment between ground- and space-based data. We did not use the broadband $4170 \AA$ continuum channel for subsequent analyses since the ROSA G-band data originate very close to the continuum level ( $\sim 75 \mathrm{~km}$ height difference; Jess et al. 2012b) and have a better signal-to-noise ratio than the ROSA $4170 \AA$ continuum.

\section{ANALYSIS AND RESULTS}

Active region NOAA 11366 comprises a circularly symmetric sunspot with fanlike loop structures visible on one side in the corresponding coronal channels (see Figure 1). A time- lapse movie of the region, as seen through the AIA $171 \AA$ filter (available online), clearly shows outwardly propagating waves in all of the fan loops. Figure 1 indicates a number of preselected regions of interest, labeled as "A," "B," and "C," with the corresponding light curves extracted from location "A" detailed in Figure 2. In this figure, the top panel displays the original light curve (after binning over $3 \times 3$ pixel $^{2}$ ), where an oscillation of $\approx 3$ minutes is visible along with other longerperiod components. The overplotted dotted line follows the low-frequency background trend, which is subtracted to filter such longer periods, with the resultant displayed in the middle panel. The bottom panel displays the reconstructed light curve obtained following Fourier filtration, allowing only a narrow band ( $\pm 50 \mathrm{~s}$ full width) of frequencies around three minutes. All light curves show the persistent presence of three-minute oscillations throughout the duration of the observing sequence. An interesting aspect to note here is the amplitude of the oscillations, which appears to increase and decrease over time. This feature is visible in all the light curves, hence ruling out the possibility that these are artifacts of the applied Fourier filters. We also observed this behavior at other locations along the wave propagation path.

The observed oscillations are similar to those previously studied by many authors (e.g., De Moortel et al. 2002; Marsh \& Walsh 2006; Jess et al. 2012a; Krishna Prasad et al. 2012, 2014, to name but a few), in fanlike loop structures. There has been a debate on whether these oscillations are due to waves or high-speed quasi-periodic upflows (De Moortel \& Nakariakov 2012), but it is widely believed that the three-minute sunspot oscillations (as presented here) are the signature of propagating slow magnetoacoustic waves. The disappearance of the oscillations after a certain length along the structure further confirms their propagating nature, and with temporal variations in amplitude observed along their full propagation path, such modulation may be a property of the source itself. In fact, similar modulations were found in sub-coronal sunspot oscillations by Beckers \& Schultz (1972), Gurman et al. (1982), Lites (1984), Thomas et al. (1987), Fludra (2001), Marsh \& Walsh (2006), and Centeno et al. (2006, 2009). Thus, an important aspect is to try and identify the wave source by tracking these oscillations along the loop to its base, and determining what feature(s) and atmospheric height(s) modulate the observed wave trains. It appears that the current fan loop is rooted in the sunspot umbra, which explains the prevalence of three-minute oscillations, and through visual inspection this loop appears to be terminated at the location marked "B" in Figure 1. Therefore, we constructed similar Fourier-filtered light curves from location "B" near the coronal footpoint of the loop (marked in Figure 1) in all channels representing different layers of the solar atmosphere, with the resulting light curves displayed in Figure 3. Although it is generally assumed that magnetic field lines in the umbral region are mostly vertical, an important aspect to consider is whether location " $\mathrm{B}$ " corresponds to the same magnetic feature when observed in all AIA channels. To determine the answer, we used the vector magnetograms from this region and employed nonlinear force-free field extrapolations (Guo et al. 2012) to reveal that the central pixel position of location "B" laterally shifts by less than 3 AIA pixels over a height of $3600 \mathrm{~km}$ above the photosphere, thus justifying our choice of a $3 \times 3$ pixel $^{2}$ binning region for all channels. For ROSA and HARDcam channels, we used equivalent macro-pixels to 

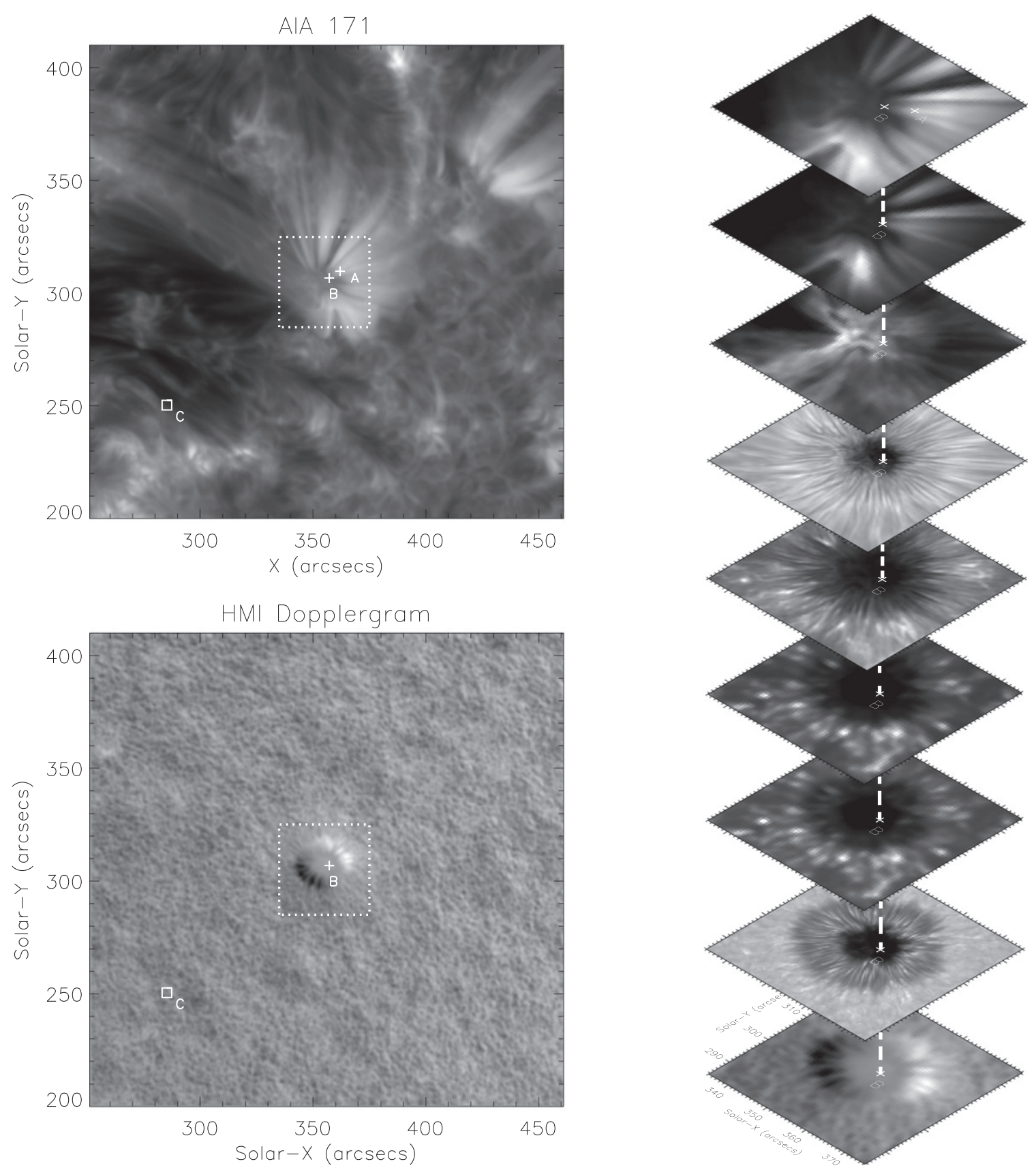

Figure 1. Left: active region NOAA 11366 and its surroundings observed in the AIA $171 \AA$ channel (top) and an HMI Dopplergram (bottom). The dotted box encloses the subfield region shown as a series of stacked images on the right. Right: the sunspot as seen in different imaging channels: (from bottom to top) HMI Dopplergram, ROSA G-band, AIA $1700 \AA$, AIA $1600 \AA$, ROSA Ca II K, HARDcam H $\alpha$, AIA 304 A, AIA 131 A, and AIA 171 A. Locations labeled "A," "B," and "C" are also marked, which are used to study wave propagation in subsequent figures. A time-lapse movie of the active region in AIA $171 \AA$ channel can be found online.

(An animation of this figure is available.)

construct the corresponding light curves. The AIA $171 \AA$ light curve from location "A" is also shown in this figure for comparison. Interestingly, the dominant oscillation period at location " $\mathrm{B}$ " is $\approx 5$ minutes in the photospheric channels, even within the umbra, which is similar to that reported earlier by several authors (Bhatnagar et al. 1972; Soltau et al. 1976; Thomas et al. 1981). The normalized Fourier power spectra generated from the corresponding original light curves are shown in left panels of Figure 3, where the numbers listed in each panel represent the peak periodicity value in seconds. The positions of the three-minute and five-minute periods are marked by vertical dashed red lines for comparison. It is evident that the oscillation power peaks near three minutes for formation heights above that of the AIA 1700 and $1600 \AA$ channels, which form near the temperature minimum region, before shifting to periodicities of $\approx 5$ minutes in lower atmospheric regions. The ROSA G-band and HMI Dopplergrams form approximately $100 \mathrm{~km}$ above the photosphere (Fleck et al. 2011; Jess et al. 2012b). It is noted that $\approx 5$ minute peaks in G-band imaging and HMI Dopplergrams are accompanied by 


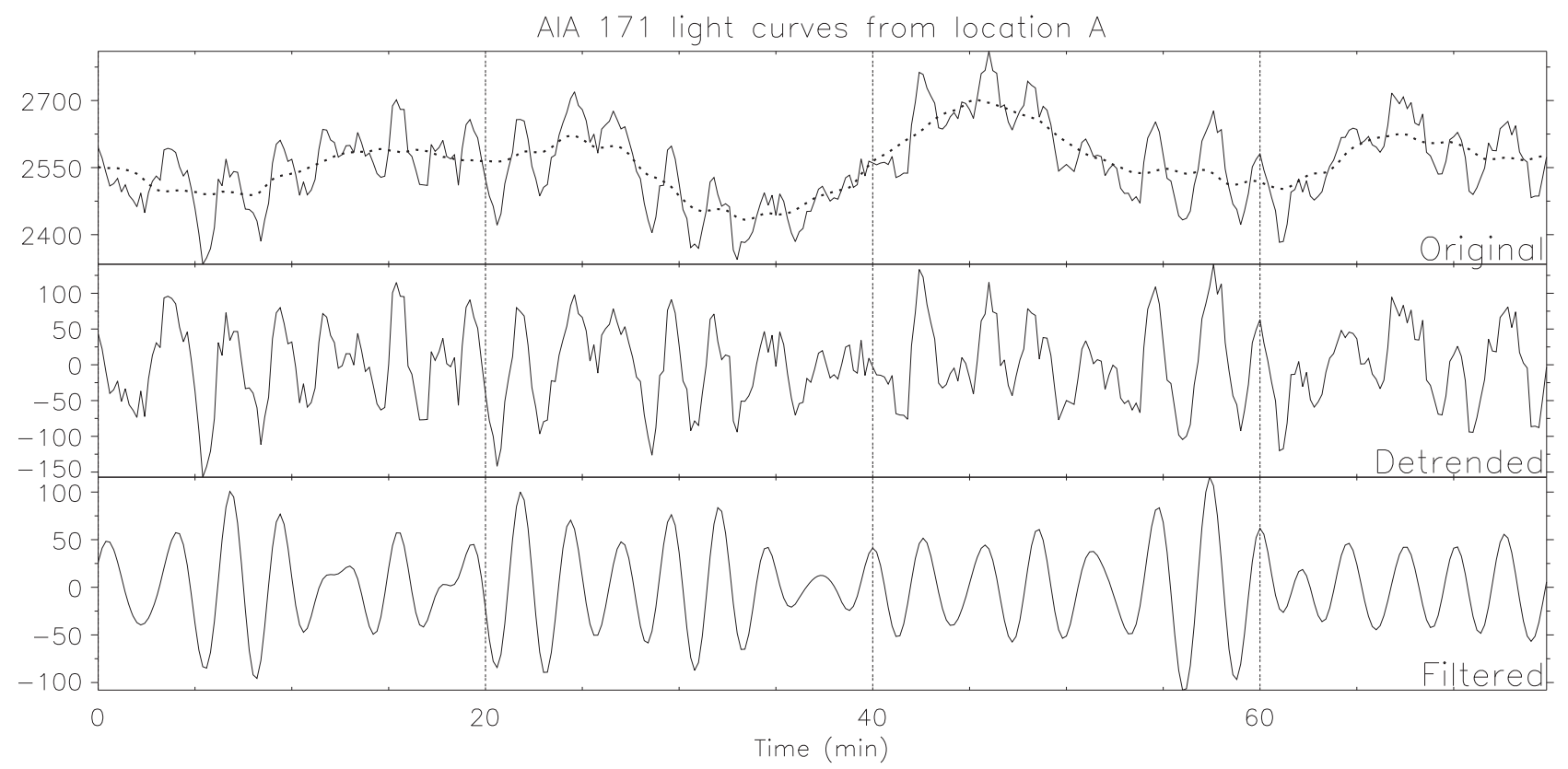

Figure 2. Light curves from location "A" (see Figure 1) as captured by the AIA $171 \AA$ channel. The top panel displays the original light curve after $3 \times 3$ pixel ${ }^{2}$ binning. The low-frequency background trend is overplotted using a dotted line, which is subsequently subtracted from the original time series to produce the detrended light curve shown in the middle panel. The bottom panel displays the reconstructed light curve following narrowband Fourier filtration around three minutes.

multiple closely spaced peaks (similar to those at $\approx 3$ minutes in the other channels), yet there is no significant enhancement at three minutes in these channels. Therefore, to accommodate this frequency shift, the reconstructed light curves displayed in Figure 3 are Fourier-filtered around five minutes for these two photospheric channels. The frequency band used in each channel to produce the filtered light curves is marked in gray over the corresponding power spectra. Furthermore, to check the nature of propagation of these oscillations from the photosphere to the corona, we estimated phase angles $(\phi)$ for the light curves at location "B," taking information from the HMI Dopplergram, AIA $1700 \AA$, and AIA $304 \AA$ channels as reference points. The obtained values are listed in Figure 3. Some channels cannot be used as a reference for photospheric and chromospheric propagation since the periodicities are different. As the observations enter an optically thin regime, the AIA $304 \AA$ channel is chosen as a reference to indicate the coronal propagation of the waves.

From Figure 3 it is clear that all light curves show a similar modulation in amplitude with time, indicating a possible connection between the five-minute photospheric oscillations and their slow magnetoacoustic counterparts observed in the corona. To extend this further and check the possible connection with photospheric $p$-modes, we identified a location (marked as $\mathrm{C}$ in Figure 1) outside the sunspot where the average absolute magnetic field strength is $<20 \mathrm{G}$ and constructed a similarly Fourier-filtered time series corresponding to that location. This light curve is shown in the bottom right panel of Figure 3 and, interestingly, also displays a similar modulation in its oscillatory amplitude. In order to quantify the observed variation in amplitude, we employed wavelet analysis over the filtered light curves and extracted the amplitude variation (i.e., correlated with the wavelet power) as a function of time. The obtained amplitudes are shown in Figure 4. By applying wavelet analysis to the obtained amplitudes, we estimated the dominant period at which the amplitudes vary.
This value is listed at the bottom left corner of each panel in Figure 4. The amplitude fluctuations appear to have a reciprocating nature with mean values on the order of 20-27 minutes. It may be noted that since the amplitudes are extracted from the narrowband Fourier-filtered light curves, the observed fluctuations purely represent the variations in amplitude of three (or five) minute oscillations and thus have distinguished themselves from the the usually present longperiod sunspot oscillations (see Figure 2, top panel). To verify if other loops in the coronal fan system behave in a similar way, we performed identical analysis at all pixel locations in the AIA $171 \AA$ channel that displayed prominent three-minute oscillations ( $\sim 600$ pixel locations), as well as all pixel locations in the HMI Dopplergrams that contained average absolute magnetic field strengths $<20 \mathrm{G}$ (over 12,000 pixel locations), and calculated their corresponding amplitude modulation periods. The results are displayed in a histogram plot shown in Figure 5, which reveals peak modulation periods of 27.0 minutes and 29.1 minutes (modal values) in the HMI and AIA channels, respectively, thus strengthening our hypothesis that the global $p$-mode oscillations and the propagating waves found in coronal loop systems are related.

\section{DISCUSSION AND CONCLUSIONS}

Propagating slow magnetoacoustic waves observed in fanlike loop structures are found to exhibit temporal variations in their amplitude. We employed multi-wavelength and multiinstrument data to track the oscillations along the length of the loop structure. Similar modulations in the wave amplitudes are observed at all atmospheric heights, including at the base of the photosphere. The amplitude fluctuations appear to be modulated with a periodicity on the order of 20-27 minutes across all bandpasses. Despite the fan loops being anchored in the sunspot umbra, the dominant oscillation period at its base shifts from three to five minutes close to the photosphere, while the 


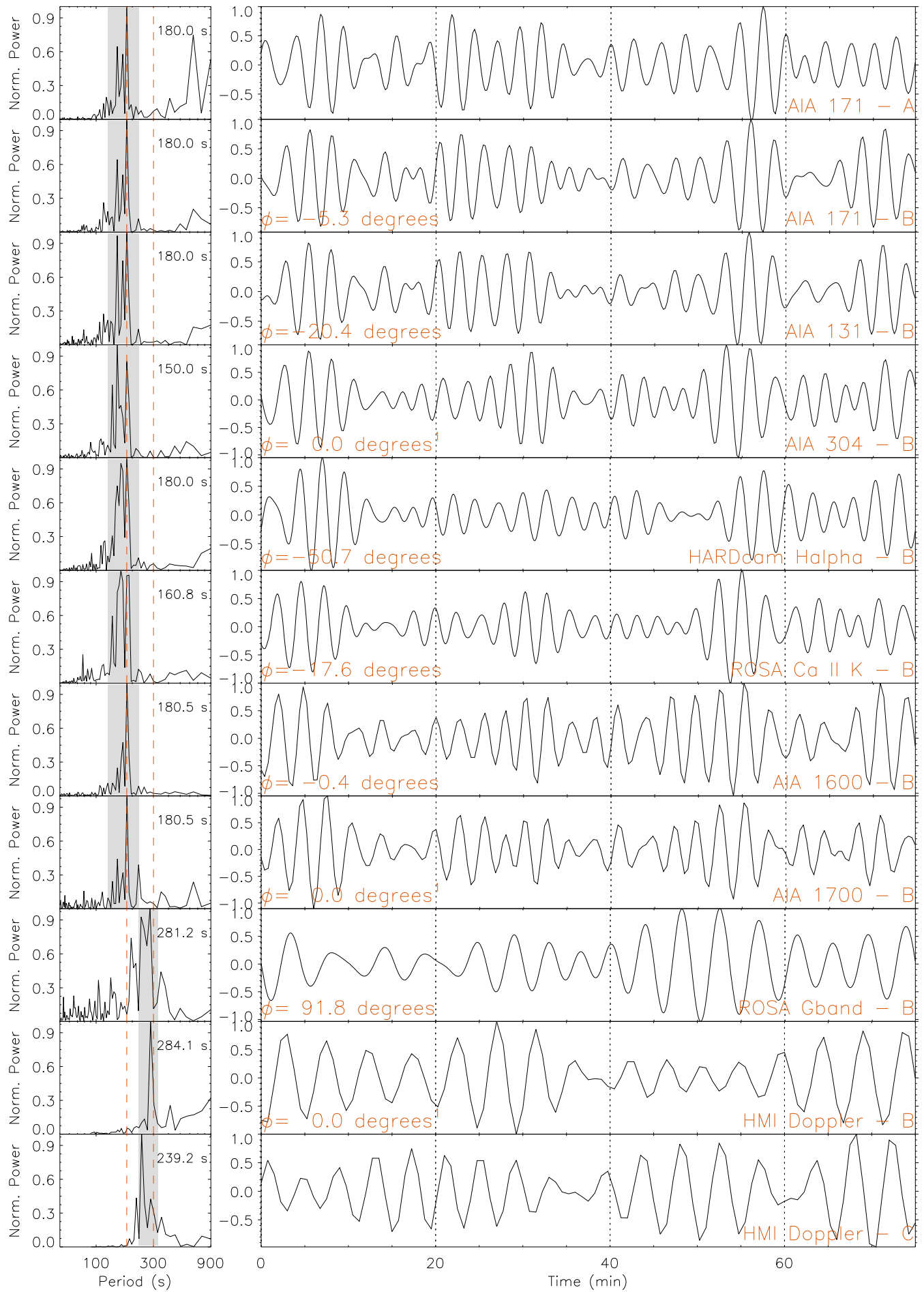

Figure 3. Left: normalized Fourier power spectra of original light curves from different $S D O$ and ROSA/HARDcam channels. The peak periodicity identified is listed in each plot in seconds. The two vertical dashed red lines mark the locations of three-minute and five-minute periodicities. Regions in gray denote the corresponding frequency band used to produce the filtered light curves shown on the right. Right: Fourier-filtered light curves from the footpoint of the fan loop (location "B" in Figure 1), plotted as a function of atmospheric height. An AIA $171 \AA$ light curve from location "A" and an HMI Dopplergram time series from location "C" are also shown for comparison. The obtained phase angles $(\phi)$ for the light curves at location B, taking the HMI Dopplergram, AIA $1700 \AA$, and AIA $304 \AA$ channels as a reference (indicated by superscript " 1 ") are also listed.

amplitude modulation period remains the same. The fiveminute Doppler oscillations from a non-magnetic region outside the active region also show similar variations in wave amplitude across the same period range. This behavior is also observed in other parts of the fan loop system, which show a good correlation in amplitude modulation periods with that of non-magnetic regions outside the sunspot in HMI Dopplergrams. These results highlight a possible connection between the photospheric $p$-modes and the propagating slow magnetoacoustic waves observed in the corona.

Amplitude modulation has previously been observed in sunspot oscillations by several authors (Beckers \& 


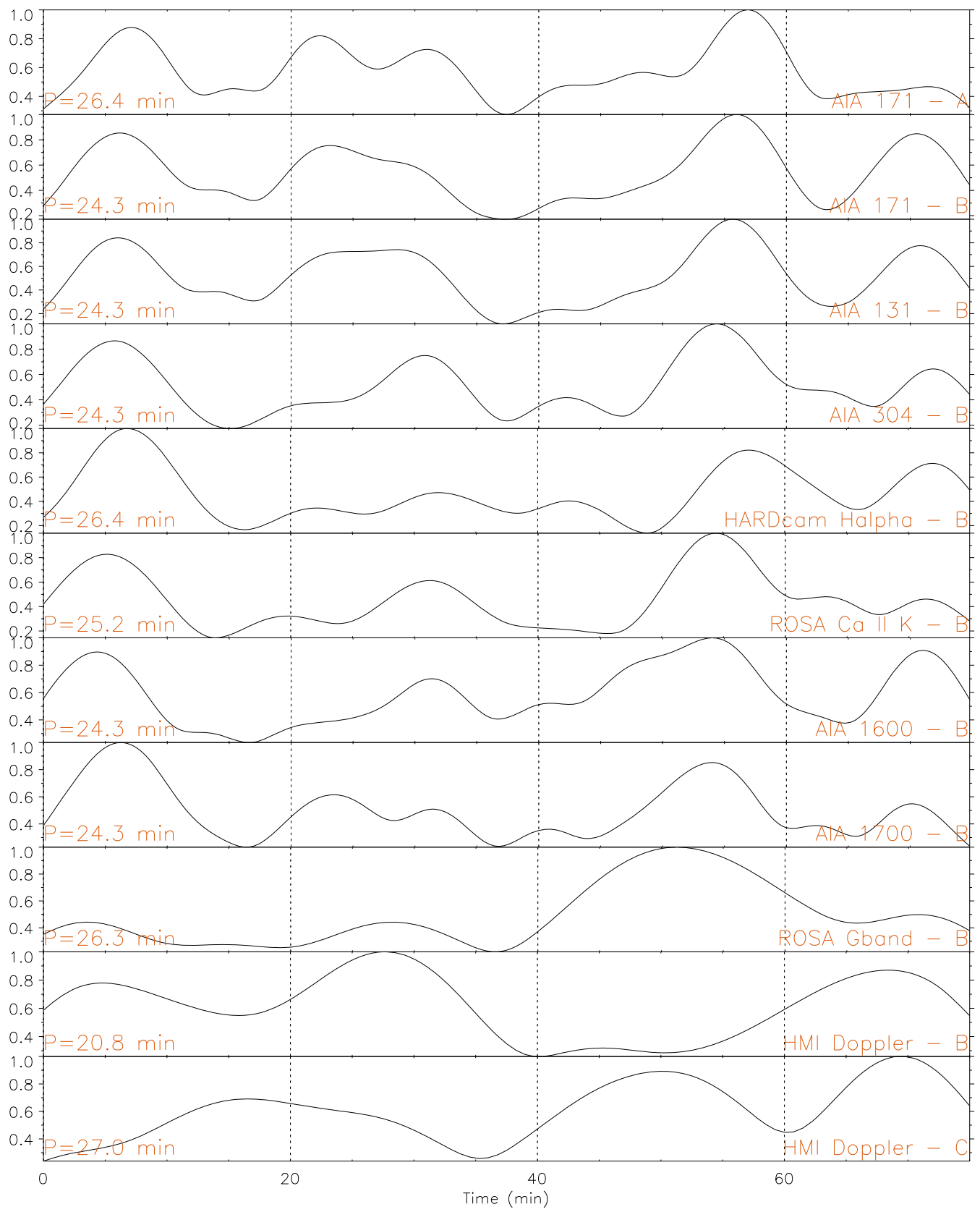

Figure 4. Oscillations amplitude variations, extracted from the Fourier-filtered light curves shown in Figure 3, are displayed in ascending atmospheric height order. An estimate of the timescales over which each amplitude modulation occurs is listed for each panel.

Schultz 1972; Gurman et al. 1982; Lites 1984; Thomas et al. 1987; Fludra 2001; Centeno et al. 2006, 2009; Marsh \& Walsh 2006), and it has been suggested that their existence is caused by a number of closely spaced frequencies leading to a beat phenomenon. The formation of multiple closely spaced frequencies is explained by resonant filter theories (Zhukov 2005), with Marsh et al. (2008) employing a Bayesian model to resolve four closely spaced frequencies in the transition region oscillations that demonstrated amplitude modulations. They identified that the frequencies were part of the global $p$-mode spectrum and suggested a link between the photosphere and the upper atmosphere. Indeed, we find multiple closely spaced peaks in the power spectra of oscillations detected over all atmospheric heights (left panels of Figure 3), which helps explain the amplitude modulations and reveals the propagation of photospheric $p$-modes up to coronal heights. Furthermore, the negative phase angles obtained in the chromospheric and coronal channels also suggest the upward propagation of waves. The nearly $90^{\circ}$ phase difference observed between the G-band intensities and the HMI Doppler velocities, which originate at approximately the same atmospheric height $(\approx 100 \mathrm{~km})$, might probably indicate some reflection at the temperature minimum region.

As suggested by many theoretical models (e.g., Hindman \& Jain 2008; Jain et al. 2014), the photospheric $p$-modes are likely the source of propagating slow magnetoacoustic waves in sunspots that are guided up to coronal heights along the strong umbral field lines, permeating coronal fans before becoming dissipated. Similarly, those upwardly propagating magnetoacoustic waves that travel along more inclined fields 


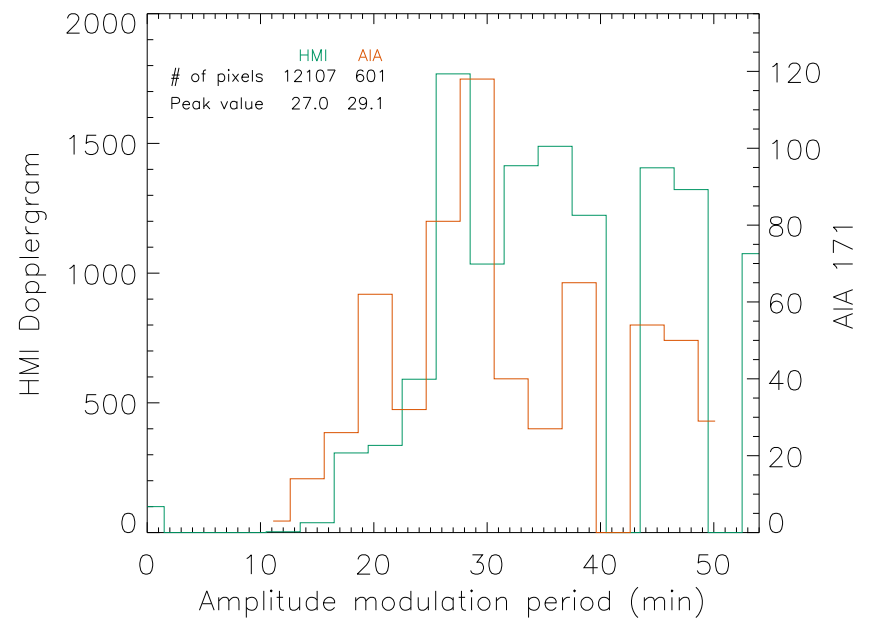

Figure 5. Histograms of amplitude modulation period from all the pixels in AIA 171 that display dominant three-minute oscillations and all the pixels in HMI Dopplergram that have magnetic field strength $<20 \mathrm{G}$.

(e.g., such as those toward the umbra/penumbra boundary) end up as running penumbral waves in the chromosphere (e.g., Kobanov et al. 2006; Jess et al. 2013). The shift in oscillation period from five to three minutes in the chromosphere can either be due to the creation of a chromospheric resonance cavity (Zhugzhda et al. 1983) or a result of the natural resonant excitation at the atmospheric cutoff frequency that is higher within the upper photosphere (Fleck \& Schmitz 1991) or due to a simple interplay between the maximum power and the cutoff frequency (Khomenko \& Collados 2015).

The authors thank the referee for useful comments. D.B.J. would like to thank STFC for an Ernest Rutherford Fellowship, in addition to a dedicated standard grant that allowed this project to be undertaken. The AIA and HMI data used here are courtesy of NASA/SDO and the AIA and HMI science teams.

Facilities: Dunn (ROSA, HARDcam), SDO (AIA, HMI).

\section{REFERENCES}

Abdelatif, T. E., Lites, B. W., \& Thomas, J. H. 1984, in Small-Scale Dynamical Processes in Quiet Stellar Atmospheres, ed. S. L. Keil (Sunspot, NM: National Solar Observatory), 141

Beckers, J. M., \& Schultz, R. B. 1972, SoPh, 27, 61

Beckers, J. M., \& Tallant, P. E. 1969, SoPh, 7, 351
Bellot Rubio, L. R., Collados, M., Ruiz Cobo, B., \& Rodríguez Hidalgo, I. 2000, ApJ, 534, 989

Bhatnagar, A., Livingston, W. C., \& Harvey, J. W. 1972, SoPh, 27, 80

Bloomfield, D. S., Lagg, A., \& Solanki, S. K. 2007, ApJ, 671, 1005

Bogdan, T. J., \& Judge, P. G. 2006, RSPTA, 364, 313

Brisken, W. F., \& Zirin, H. 1997, ApJ, 478, 814

Centeno, R., Collados, M., \& Trujillo Bueno, J. 2006, ApJ, 640, 1153

Centeno, R., Collados, M., \& Trujillo Bueno, J. 2009, ApJ, 692, 1211

Christopoulou, E. B., Georgakilas, A. A., \& Koutchmy, S. 2000, A\&A, 354,305

Christopoulou, E. B., Georgakilas, A. A., \& Koutchmy, S. 2001, A\&A, 375,617

De Moortel, I., \& Hood, A. W. 2003, A\&A, 408, 755

De Moortel, I., Ireland, J., Hood, A. W., \& Walsh, R. W. 2002, A\&A, 387, L13

De Moortel, I., \& Nakariakov, V. M. 2012, RSPTA, 370, 3193

Fleck, B., Couvidat, S., \& Straus, T. 2011, SoPh, 271, 27

Fleck, B., \& Schmitz, F. 1991, A\&A, 250, 235

Fludra, A. 2001, A\&A, 368, 639

Giovanelli, R. G. 1972, SoPh, 27, 71

Guo, Y., Ding, M. D., Liu, Y., et al. 2012, ApJ, 760, 47

Gurman, J. B., Leibacher, J. W., Shine, R. A., Woodgate, B. E., \& Henze, W. 1982, ApJ, 253, 939

Henriques, V. M. J., Scullion, E., Mathioudakis, M., et al. 2015, A\&A, 574, A131

Hindman, B. W., \& Jain, R. 2008, ApJ, 677, 769

Jain, R., Gascoyne, A., Hindman, B. W., \& Greer, B. 2014, ApJ, 796, 72

Jess, D. B., De Moortel, I., Mathioudakis, M., et al. 2012a, ApJ, 757, 160

Jess, D. B., Mathioudakis, M., Christian, D. J., et al. 2010, SoPh, 261, 363

Jess, D. B., Morton, R. J., Verth, G., et al. 2015, SSRv, 190, 103

Jess, D. B., Reznikova, V. E., Van Doorsselaere, T., Keys, P. H., \& Mackay, D. H. 2013, ApJ, 779, 168

Jess, D. B., Shelyag, S., Mathioudakis, M., et al. 2012b, ApJ, 746, 183

Khomenko, E., \& Collados, M. 2015, LRSP, in press

Kobanov, N. I., Kolobov, D. Y., \& Makarchik, D. V. 2006, SoPh, 238, 231

Krishna Prasad, S., Banerjee, D., \& Singh, J. 2012, SoPh, 281, 67

Krishna Prasad, S., Banerjee, D., \& van Doorsselaere, T. 2014, ApJ, 789, 118

Landgraf, V. 1997, AN, 318, 129

Lemen, J. R., Title, A. M., Akin, D. J., et al. 2012, SoPh, 275, 17

Lites, B. W. 1984, ApJ, 277, 874

Löhner-Böttcher, J., \& Bello González, N. 2015, A\&A, 580, 53

Marsh, M. S., Ireland, J., \& Kucera, T. 2008, ApJ, 681, 672

Marsh, M. S., \& Walsh, R. W. 2006, ApJ, 643, 540

Pesnell, W. D., Thompson, B. J., \& Chamberlin, P. C. 2012, SoPh, 275, 3

Reznikova, V. E., Shibasaki, K., Sych, R. A., \& Nakariakov, V. M. 2012, ApJ, 746, 119

Rouppe van der Voort, L. H. M., Rutten, R. J., Sütterlin, P., Sloover, P. J., \& Krijger, J. M. 2003, A\&A, 403, 277

Schou, J., Scherrer, P. H., Bush, R. I., et al. 2012, SoPh, 275, 229

Soltau, D., Schroeter, E. H., \& Woehl, H. 1976, A\&A, 50, 367

Thomas, J. H., Cram, L. E., \& Nye, A. H. 1981, BAAS, 13, 858

Thomas, J. H., Lites, B. W., Gurman, J. B., \& Ladd, E. F. 1987, ApJ, 312, 457

Zhugzhda, I. D., Locans, V., \& Staude, J. 1983, SoPh, 82, 369

Zhukov, V. I. 2005, A\&A, 433, 1127

Zirin, H., \& Stein, A. 1972, ApJL, 178, L85 